\title{
Regularizations of general singular integral operators
}

\author{
Constanze Liaw and Sergei Treil
}

Abstract. In the theory of singular integral operators significant effort is often required to rigorously define such an operator. This is due to the fact that the kernels of such operators are not locally integrable on the diagonal $s=t$, so the integral formally defining the operator $T$ or its bilinear form $\langle T f, g\rangle$ is not well defined (the integrand in not in $L^{1}$ ) even for "nice" $f$ and $g$. However, since the kernel only has singularities on the "diagonal" $s=t$, the bilinear form $\langle T f, g\rangle$ is well defined, say, for bounded compactly supported functions with separated supports.

One of the standard ways to interpret the boundedness of a singular integral operators is to consider the regularized kernel

$$
K_{\varepsilon}(s, t)=K(s, t) m((s-t) / \varepsilon),
$$

where the cut-off function $m$ is 0 in a neighborhood of the origin, so the integral operators $T_{\varepsilon}$ with kernel $K_{\varepsilon}$ are well defined (at least on a dense set). Then instead of asking about the boundedness of the operator $T$, which is not well defined, one can ask about uniform boundedness (in $\varepsilon$ ) of the regularized operators $T_{\varepsilon}$.

For the standard regularizations one usually considers truncated operators $T_{\varepsilon}$ with $m(s)=\mathbf{1}_{[1, \infty)}(|s|)$, although smooth cut-off functions were also considered in the literature.

The main result of the paper is that for a wide class of singular integral operators (including the classical Calderón-Zygmund operators in nonhomogeneous two weight settings), the so called restricted $L^{p}$ boundedness, i.e., the uniform estimate

$$
|\langle T f, g\rangle| \leq C\|f\|_{p}\|g\|_{p^{\prime}}
$$

for bounded compactly supported $f$ and $g$ with separated supports implies the uniform $L^{p}$-boundedness of regularized operators $T_{\varepsilon}$ for any reasonable choice of smooth cut-off function $m$. For example, any $m \in C^{\infty}\left(\mathbb{R}^{N}\right), m \equiv 0$ in a neighborhood of 0 , and such that $1-m$ is compactly supported would work.

Mathematics Subject Classification (2010): 42B20.

Keywords: Singular integral operator, regularization, truncation, Calderón-Zygmund operator, restricted boundedness, two weight Muckenhoupt condition. 
If the kernel $K$ satisfies some additional assumptions (which are satisfied for classical singular integral operators like the Hilbert transform, Cauchy transform, Ahlfors-Beurling transform, and generalized Riesz transforms), then the restricted $L^{p}$ boundedness also implies the uniform $L^{p}$ boundedness of the classical truncated operators $T_{\varepsilon}\left(m(s)=\mathbf{1}_{[1, \infty)}(|s|)\right)$.

\section{Introduction}

\subsection{Preliminaries}

Generally, a singular integral operator is understood as an operator $T$ on $L^{2}(\mu)$ (or on $L^{p}(\mu)$ ) that is given formally by

$$
T f(s)=\int K(s, t) f(t) d \mu(t)
$$

where the kernel $K(s, t)$ is singular near $s=t$, i.e., $K(s, \cdot)$ and $K(\cdot, t)$ are not in $L_{\text {loc }}^{1}$ near the singularity. This means that the above integral is not defined even for the simplest functions $f$ (which explains the word formally above), and the question of how to interpret this expression immediately arises.

In simple cases the interpretation is quite easy. For example, if $T$ is the classical Hilbert transform on the real line $\left(K(s, t)=\pi^{-1}(s-t)^{-1}, \mu\right.$ is the Lebesgue measure on $\mathbb{R})$, it is an easy exercise to show that for a compactly supported smooth function $f$ the integral (1.1) exists in the sense of principal value, i.e., that

$$
\lim _{\alpha \rightarrow 0+} \int_{|s-t|>\alpha} \frac{f(t)}{s-t} d t
$$

exists (for all $s$, provided that $f$ is $C^{1}$ and compactly supported). Thus, the operator $T$ is well defined on a dense set, and if one proves $L^{2}$ (or $L^{p}$ ) bounds on this set, the operator extends by continuity to all $L^{2}$ (resp. $L^{p}$ ). For the Hilbert transform the $L^{p}$ estimates are a classical and well known result, so the Hilbert transform is a well defined bounded operator in $L^{p}, p \in(1, \infty){ }^{1}$

Such a naïve approach also works for other "nice" classical singular integral operators, like Riesz transforms in $\mathbb{R}^{n}$. However, the situation becomes more complicated if one considers more general measures and/or kernels: the existence of principal values in such situations is far from trivial, and usually requires a lot of effort.

So, given a (formal) singular integral operator, how can one define it and investigate whether it is bounded in $L^{p}$ ? One of the standard approaches in the general situation is to consider truncated operators $T_{\varepsilon}$,

$$
T_{\varepsilon} f(s)=\int_{|s-t|>\varepsilon} K(s, t) f(t) d \mu(t),
$$

\footnotetext{
${ }^{1}$ It is also known, although significantly harder to prove, that the principal value (1.2) exists a.e. for all compactly supported $L^{1}$ functions $f$, which immediately implies the existence of the principal value for all $f \in L^{p}, p \in(1, \infty)$.
} 
which are well defined for compactly supported $f$ if one assumes, for example, that $K$ is locally bounded off the "diagonal" $s=t$. In this case, the boundedness in $L^{p}$ is defined as the uniform boundedness of the truncated operators $T_{\varepsilon}$; if the operators $T_{\varepsilon}$ are uniformly bounded, one can then take a limit point (in the weak operator topology) of $T_{\varepsilon}, \varepsilon \rightarrow 0$, as the corresponding singular integral operator $T$. Note that the weak limit point does not have to be unique.

Moreover, if the operators $T_{\varepsilon}$ are uniformly bounded, then it is often possible to prove the existence of principal values (at least for $f$ in a dense set), so one can define the singular integral operator in a natural fashion.

Instead of truncations, one can also consider smooth regularizations of the kernel $K$. For example, for the Hilbert transform, it is very natural to move to the complex plane and consider operators $T_{\varepsilon}$,

$$
T_{\varepsilon} f(s)=\frac{1}{\pi} \int_{\mathbb{R}} \frac{f(t)}{s-t+i \varepsilon} d t .
$$

Further, there is an alternative, "axiomatic" way of defining a singular integral operator with kernel $K$, see for example [1]. Namely, we assume that we are given an operator, or, more precisely its bilinear form $\langle T f, g\rangle$, well defined on some smaller set (the Schwartz class or the class of $C^{\infty}$ functions with compact support are often used). The statement that $T$ is an integral operator with kernel $K$ means simply that

$$
\langle T f, g\rangle=\int K(s, t) f(t) g(s) d \mu(t) d \mu(s)
$$

for $f$ and $g$ with separated compact supports (so the above integral is well defined). In many cases it was shown that if the operator $T$ is bounded in $L^{p}$, then the truncated operators $T_{\varepsilon}$ are uniformly bounded as well. Note, that in the above abstract approach we require some kind of a priori bounds on the operator, because we assume that its bilinear form is well defined on some smaller space.

Let us also mention, that in the theory of Calderón-Zygmund operators (see the definition of a Calderón-Zygmund operator below), if the kernel $K$ is antisymmetric, $K(s, t)=-K(t, s)$, there is a canonical way to interpret the operator without any a priori boundedness assumptions, see for example [1] for the homogeneous case and [7] for the non-homogeneous case.

Namely, antisymmetry means that formally $T^{*}=-T$, so $\langle T f, g\rangle=-\langle f, T g\rangle$, so (again formally)

$$
\begin{aligned}
\langle T f, g\rangle & =\int K(s, t) f(t) g(s) d \mu(t) d \mu(s)=-\int K(s, t) f(s) g(t) d \mu(t) d \mu(s) \\
& =\frac{1}{2} \int K(s, t)[f(t) g(s)-f(s) g(t)] d \mu(t) d \mu(s) .
\end{aligned}
$$

If $K$ is a Calderón-Zygmund kernel of dimension $d$ in $\mathbb{R}^{N}$, then by the definition $|K(s, t)| \leq C|s-t|^{-d}$. Therefore if $f$ and $g$ are compactly supported $C^{1}$ functions, then the integrand in (1.4) can be estimated by $C|s-t|^{-d+1}$.

For such kernels it is usually assumed that the measure satisfies the condition $\mu\left(\left\{x:\left|x-x_{0}\right|<r\right\}\right) \leq C r^{d}$ for all $x_{0}$ and $r$ (this condition is usually necessary for 
the $L^{2}$ boundedness of classical Calderón-Zygmund operators of dimension $d$, like Cauchy transform in $\mathbb{C})$. But for such measures $\int_{Q}|s-t|^{-d+1} d \mu(s) d \mu(t)<\infty$ for any compact $Q$, so $\langle T f, g\rangle$ is well defined for $f, g \in C_{0}^{1}$.

\subsection{Description of the main results}

The main result of the paper can be stated in one sentence as "the situation with interpretation of singular integral operators is much simpler than it seems; to investigate the boundedness one only needs to study an elementary and well defined restricted bilinear form".

The main idea is embarrassingly simple, and we should be ashamed that we did not arrive at it much earlier, although some preliminary results in this direction were obtained by us in [6], and in thesis of the first author. In our defense we can only say that this idea was overlooked by generations of harmonic analysts before us.

Let us describe the main results in more detail. We will need some definitions.

Definition 1.1. Let Radon measures $\mu$ and $\nu$ in $\mathbb{R}^{N}$ be fixed. A singular kernel in $\mathbb{R}^{N}$ (with respect to the measures $\mu$ and $\nu$ ) is a $\mu \times \nu$-measurable function $K$ on $\mathbb{R}^{N} \times \mathbb{R}^{N}$, which is locally $L^{2}(\mu \times \nu)$ off the diagonal $\left\{(s, t) \in \mathbb{R}^{N} \times \mathbb{R}^{N}: s=t\right\}$.

We say that the singular kernel $K$ is a singular kernel of order $d$ if the kernel

$$
\widetilde{K}(s, t)= \begin{cases}K(s, t)|s-t|^{d}, & s \neq t, \\ 0, & s=t\end{cases}
$$

is locally $L^{2}(\mu \times \nu)$. Note, that for any singular kernel on $\mathbb{R}^{N}$ the kernel $\widetilde{K}$ is locally $L^{2}(\mu \times \nu)$ off the diagonal, so one only needs to check this condition on the diagonal.

For a singular kernel $K$ in $\mathbb{R}^{N}$ (with respect to Radon measures $\mu$ and $\nu$ ) the expression

$$
\langle T f, g\rangle=\int K(s, t) f(t) g(s) d \mu(t) d \nu(s)
$$

is well defined for all Borel measurable bounded functions $f, g$ with separated compact supports $(\operatorname{dist}(\operatorname{supp} f, \operatorname{supp} g)>0)$.

Definition 1.2. Let $p \in(1, \infty)$ and Radon measures $\mu$ and $\nu$ on $\mathbb{R}^{N}$ be fixed. Let $K$ be a singular kernel in $\mathbb{R}^{N}$. We say that the formal singular integral operator with the kernel $K$ is restrictedly bounded in $L^{p}$ (i.e., as an operator $T: L^{p}(\mu) \rightarrow$ $\left.L^{p}(\nu)\right)$ if

$$
\left|\int K(s, t) f(t) g(s) d \mu(t) d \nu(s)\right| \leq C\|f\|_{L^{p}(\mu)}\|g\|_{L^{p^{\prime}(\nu)}}, \quad 1 / p+1 / p^{\prime}=1
$$

for all bounded $f, g$ with separated compact supports. Sometimes, abusing the language, we will just say that the kernel $K$ is restrictedly $L^{p}$ bounded with bound $C$. The least constant $C$ in (1.5) (with $p, \mu$ and $\nu$ fixed) is called the restricted norm of $K$. 
It is easy to check that for any fixed $p, \mu, \nu$ the restricted norm is a (semi)norm on the set of singular kernels.

Remark. For fixed measures $\mu$ and $\nu$ one can always assume that the kernel $K$ is defined only a.e. with respect to the measure $\mu \times \nu$. It is not hard to show that if the measures $\mu$ and $\nu$ do not have common atoms (which we usually assume), then the diagonal $\left\{(s, s): s \in \mathbb{R}^{N}\right\}$ of $\mathbb{R}^{N} \times \mathbb{R}^{N}$ has $\mu \times \nu$ measure 0 , so the values of $K$ on the diagonal do not have to be specified.

Our first main result is that there are many families of smooth mollifying multipliers $M_{\varepsilon}(s, t)$, such that

(i) $M_{\varepsilon}(s, t) \rightarrow 1$ as $\varepsilon \rightarrow 0$ uniformly on all sets $\left\{s, t \in \mathbb{R}^{N}:|s-t|>a\right\}, a>0$;

(ii) for any singular kernel $K$ (with respect to Radon measures $\mu$ and $\nu$ ) the regularized kernels $K_{\varepsilon}=K M_{\varepsilon}$ are locally in $L^{2}(\mu \times \nu)$, so the corresponding operators are well defined for bounded compactly supported functions;

(iii) if the kernel is restrictedly bounded in $L^{p}$ (i.e., if the estimate (1.5) holds for all bounded $f, g$ with separated compact supports), and the measures $\mu$ and $\nu$ do not have common atoms, then the regularized integral operators $T_{\varepsilon}$ with kernels $K_{\varepsilon}$ are uniformly (in $\varepsilon$ ) bounded as operators $L^{p}(\mu) \rightarrow L^{p}(\nu)$.

In this case, one can take the limit point $T$ (in the weak operator topology) of $T_{\varepsilon}, \varepsilon \rightarrow 0+$, as a singular integral operator with the kernel $K$. It is easy to see that

$$
\langle T f, g\rangle=\int K(s, t) f(t) g(s) d \mu(t) d \nu(s)
$$

for all bounded $f$ and $g$ with separated compact supports, so $T$ is indeed a singular integral operator with kernel $K$ in the sense of the abstract "axiomatic" approach, described above in Section 1.1.

Note that such a limit point does not need to be unique, but it is easy to show that the difference between any two bounded operators $L^{p}(\mu) \rightarrow L^{p}(\nu)$ satisfying (1.6) (with the same kernel $K$ ) is always a multiplication operator.

A simple way to construct a mollifying multiplier is to take an arbitrary $C^{\infty}$ function $m, m \equiv 0$ in a neighborhood of the origin, and such that $1-m$ has a compact support, and define $M_{\varepsilon}(s, t):=m((t-s) / \varepsilon)$. If at the origin one only requires that $|m(x)| \leq C|x|^{d}$, then the function $M_{\varepsilon}(s, t):=m((t-s) / \varepsilon)$ will regularize the kernels of order up to $d$.

Next, we will show that, under additional assumptions on the kernel $K$, the restricted boundedness of $K$ implies the uniform boundedness of the truncated operators (1.3).

And finally, we will show that under some additional assumption, the restricted boundedness implies a two weight Muckenhoupt condition, see Theorem 5.1 below for the exact statement. 


\section{Smooth regularizations of singular kernels}

\subsection{A trivial idea}

We start with the simple observation that if a kernel $K(s, t), s, t \in \mathbb{R}^{n}$ is restrictedly bounded in $L^{p}$ with a bound $C$, then for any $a \in \mathbb{R}^{N}$ the kernel

$$
K(s, t) e^{-i a \cdot t} e^{i a \cdot s}
$$

is also restrictedly bounded with the same constant. This follows from the trivial fact that a multiplication by a unimodular function is always an invertible isometry in all $L^{p}(\mu)$, and that it does not change the support.

Averaging the kernel $K(s, t) e^{-i a \cdot t} e^{i a \cdot s}$ over all $a \in \mathbb{R}^{N}$ with weight $\rho, \rho \geq 0$ and $\int_{\mathbb{R}^{N}} \rho(a) d a=1$, we get that the averaged kernel

$$
\int_{\mathbb{R}^{N}} \rho(a) K(s, t) e^{-i a \cdot t} e^{i a \cdot s} d a
$$

is also restrictedly bounded with the same constant $C$. Note, that we do not even have to assume that $\rho \geq 0$. It is sufficient to assume that $\rho \in L^{1}(d x)$ ( $L^{1}$ with respect to Lebesgue measure); in this case the averaged kernel will be bounded with constant $C\|\rho\|_{1}$, where $\|\cdot\|_{1}$ is the $L^{1}$ norm with respect to the Lebesgue measure.

One can immediately see that

$$
\int_{\mathbb{R}^{N}} \rho(a) K(s, t) e^{-i a \cdot t} e^{i a \cdot s} d a=\widehat{\rho}(t-s) K(s, t),
$$

where $\widehat{\rho}$ denotes the Fourier transform, $\widehat{\rho}(s)=\int_{\mathbb{R}^{N}} \rho(x) e^{-i s \cdot x} d x$.

We can summarize the above reasoning in the following lemma:

Lemma 2.1. Let $K$ be a restrictedly $L^{p}$ bounded kernel (i.e., estimate (1.5) holds for all bounded compactly supported functions with separated supports) with a bound $C$. Assume that $\rho \in L^{1}(d x)$ and let

$$
M=1-\widehat{\rho}, \quad M_{\varepsilon}(x):=M(x / \varepsilon) .
$$

Then the kernels $K_{\varepsilon}(s, t):=K(s, t) M_{\varepsilon}(t-s)$ are $L^{p}$ restrictedly bounded with constant $\left(1+\|\rho\|_{1}\right) C$.

Proof. The estimate for $\varepsilon=1$ was already explained above. To prove the estimate for general $\varepsilon$ need only notice that $\widehat{\rho}(s / \varepsilon)$ is the Fourier transform of the function $\varepsilon^{N} \rho(\varepsilon x)$ and

$$
\int_{\mathbb{R}^{N}}\left|\varepsilon^{N} \rho(\varepsilon x)\right| d x=\int_{\mathbb{R}^{N}}|\rho(x)| d x=\|\rho\|_{1} .
$$

Lemma 2.2. For the function $M\left(\right.$ and $\left.M_{\varepsilon}\right)$ defined in the previous lemma, the following holds:

(i) For any $a>0$, the function $M_{\varepsilon}(s) \rightarrow 1$ as $\varepsilon \rightarrow 0+$ uniformly on the set $\left\{s \in \mathbb{R}^{N},|s|>a\right\}$. 
(ii) If $\widehat{\rho} \in C^{1}$ (e.g., if $\left.\int_{\mathbb{R}^{N}}(1+|x|)|\rho(x)| d x<\infty\right)$ and if $\int_{\mathbb{R}^{N}} \rho(x) d x=1$, then for any $\varepsilon>0$

$$
M_{\varepsilon}(s)=O(|s|) \quad \text { as } s \rightarrow 0 .
$$

(iii) If $\int_{\mathbb{R}^{N}}\left(1+|x|^{2}\right)|\rho(x)| d x<\infty, \int_{\mathbb{R}^{N}} \rho(x) d x=1$, and

$$
\int_{\mathbb{R}^{N}} x_{k} \rho(x) d x=0, \quad \forall k=1,2, \ldots, N,
$$

then

$$
M_{\varepsilon}(s)=O\left(|s|^{2}\right) \quad \text { as } s \rightarrow 0 .
$$

(iv) Moreover, if $\int_{\mathbb{R}^{N}} \rho(x) d x=1$ and if $\int_{\mathbb{R}^{N}}\left(1+|x|^{k}\right)|\rho(x)| d x<\infty$ for some $k \in \mathbb{N}$, and

$$
\int_{\mathbb{R}^{N}} x^{\alpha} \rho(x) d x=0
$$

for all multi-indices $\alpha,|\alpha|<k$, then

$$
M_{\varepsilon}(s)=O\left(|s|^{k}\right) \quad \text { as } s \rightarrow 0 .
$$

Proof. The proof follows from the basic properties of the Fourier transform. We leave it as an exercise for the reader. Statement (i) follows, for example, from the Riemann-Lebesgue Lemma.

\subsection{Some examples}

Example 2.3. On the real line $\mathbb{R}$ consider the weight $\rho(x)=e^{-x} \mathbf{1}_{[0, \infty)}(x)$. For this weight $\widehat{\rho}(s)=(1+i s)^{-1}$, so the mollifying factor $M(s)=1-\widehat{\rho}(s)$ is given by

$$
M(s)=\frac{i s}{1+i s}=\frac{s}{s-i}, \quad \text { so } \quad M_{\varepsilon}(s):=M(s / \varepsilon)=\frac{s}{s-i \varepsilon} .
$$

For the Hilbert transform kernel $K(s, t)=\pi^{-1}(s-t)^{-1}$ the regularization with this mollifying factor give as the kernel

$$
K_{\varepsilon}(s, t)=\frac{1}{\pi} \cdot \frac{1}{s-t} M_{\varepsilon}(t-s)=\frac{1}{\pi} \cdot \frac{1}{s-t} \cdot \frac{t-s}{t-s-i \varepsilon}=\frac{1}{\pi} \cdot \frac{1}{s-t+i \varepsilon},
$$

which has the very natural complex analytic interpretation. That regularization is widely used in complex analysis, and our investigation of this regularization in [6] lead us to the main idea of this paper.

Example 2.4. Define the weight $\rho$ on $\mathbb{R}^{N}$ by $\rho(x)=(2 \pi)^{-N / 2} e^{-|x|^{2} / 2}$. The Fourier transform of $\rho$ is given by $\widehat{\rho}(s)=e^{-|s|^{2} / 2}$, so the mollifying multiplier is $M(s)=1-e^{-|s|^{2} / 2}$. The regularized kernel $K_{\varepsilon}$ will be

$$
K_{\varepsilon}(s, t):=K(s, t)\left[1-e^{-|s-t|^{2} / 2 \varepsilon}\right] .
$$

Since $M$ has a zero of order 2 at 0 , this function will regularize singular kernels of order $d \leq 2$ (i.e., such that $|s-t|^{d} K(s, t)$ is locally bounded). 
The mollifying multiplier $M$ regularizes only singular kernels of order $d \leq 2$. To regularize kernels of higher order one can use, for example, its power $M_{\varepsilon}^{k}$, where the exponent $k$ is an integer, $k \geq N / 2$. Applying Lemma $2.1 k$ times we get that given an $L^{p}$ restrictedly bounded kernel $K$ with a bound $C$, then $K(s, t) M_{\varepsilon}(t-s)^{k}$ is also restrictedly bounded with constant $2^{k}$.

However, we neither have to do that, nor do we need to be tied to a particular regularization. Using basic facts about the Fourier transform, we can construct mollifying multipliers without explicitly defining the weight and computing its Fourier transform.

\subsection{Schur multipliers, Wiener algebra and Sobolev spaces}

Let us first introduce some definitions.

Definition 2.5. Let $p \in(1, \infty)$ and Radon measures $\mu$ and $\nu$ in $\mathbb{R}^{N}$ be fixed.

We say that a function $M$ on $\mathbb{R}^{N} \times \mathbb{R}^{N}$ is a Schur multiplier on the set of restrictedly $L^{p}$ bounded singular kernels, if the map $K \mapsto K M$ is a bounded map on this set. In other words, $M$ is a Schur multiplier on this set if, there exists a constant $C_{1}$ such that for any $L^{p}$ restrictedly bounded kernel $K$ with a bound $C$, see Definition 1.2, the kernel $M K$ is also restrictedly bounded with constant $C_{1} C$.

The best possible constant $C_{1}$ (for a fixed $p, \mu$, and $\nu$ ) is called the $\left(L^{p}, \mu, \nu\right.$ ) restricted Schur norm of $M$.

Remark. Let us explain the terminology a little bit. The Schur product $A \circ B$ of two matrices is their entrywise product, $(A \circ B)_{j, k}=a_{j, k} b_{j, k}$.

Similarly, the Schur product of two kernels, $K(s, t)$ and $M(s, t)$ is their usual product (of two functions). The special term "Schur product" is sometimes used to distinguish it from the product (composition) of the corresponding integral operators.

Let $X$ be a space of operators (like the space of bounded operators, or the Schatten-Von-Neumann class $\mathfrak{S}_{p}$ ). On the set of kernels one can introduce the norm inherited from the space $X$ of operators; the norm $\|K\|_{X}$ of a kernel is simply the norm of the corresponding integral operator in the space $X$.

A function $M$ is called a Schur multiplier for the class $X$ if the map $K \mapsto M K$ is bounded with respect to the norm $\|K\|_{X}$.

We use the same term "Schur multiplier", because our definition is very close in spirit to the classical one.

We should also mention that while our definition definitely depends on $p, \mu$ and $\nu$, the Schur multipliers we construct below will be the universal ones: they are Schur multipliers with uniform estimate on the Schur norm for all $p, \mu, \nu$, and also for all reasonable classes of operators like the bounded operators, $\mathfrak{S}_{p}$.

Recall, that the Wiener algebra $W\left(\mathbb{R}^{N}\right)$ is the set of all $f=\widehat{h}, h \in L^{1}\left(\mathbb{R}^{N}\right)$, with the norm $\|f\|_{W}=\|h\|_{1}$.

The reasoning in the beginning of this section can be summarized in the following lemma. 
Lemma 2.6. Let $M \in W=W\left(\mathbb{R}^{N}\right)$. Then the function $\widetilde{M}$ on $\mathbb{R}^{N} \times \mathbb{R}^{N}$ defined by $\widetilde{M}(s, t)=M(t-s)$ is a Schur multiplier with Schur norm at most $\|M\|_{W}$.

The next trivial and well known lemma gives a simple sufficient condition for $M \in W$.

Lemma 2.7. Let $M$ belong to the Sobolev space $W^{k, 2}\left(\mathbb{R}^{N}\right)=H^{k}\left(\mathbb{R}^{N}\right)$ (all derivatives up to order $k$ are in $\left.L^{2}\right), k>N / 2$. Then $M$ belongs to the Wiener algebra $W\left(\mathbb{R}^{N}\right)$, and $\|M\|_{W} \leq C\|M\|_{W^{k, 2}}$, where $C=C(N)$.

Proof. Let $\rho$ be the inverse Fourier transform of $M$.

The condition $M \in W^{k, 2}\left(\mathbb{R}^{N}\right)$ means that $\left(1+|x|^{k}\right) \rho \in L^{2}\left(\mathbb{R}^{N}\right)$. Then, by the Cauchy-Schwarz inequality,

$$
\begin{aligned}
\int_{\mathbb{R}^{N}}|\rho(x)| d x & \leq\left(\int_{\mathbb{R}^{N}}\left(1+|x|^{k}\right)^{-2} d x\right)^{1 / 2}\left(\int_{\mathbb{R}^{N}}\left(1+|x|^{k}\right)^{2}|\rho(x)|^{2} d x\right)^{1 / 2} \\
& \leq C(N)\|M\|_{W^{k, 2}}
\end{aligned}
$$

so $M$ belongs to the Wiener algebra.

Note that the sufficient condition $M \in W^{k, 2}$ is far from necessary: while the Wiener algebra is scale invariant, i.e., the scaling operator $S_{a}, S_{a} f(x)=f(a x)$, is an isometry in $W$, one can easily see that the operator norm $\left\|S_{a}\right\|_{W^{k, 2} \rightarrow W^{k, 2}} \rightarrow \infty$ as $a \rightarrow 0$.

The next lemma, an analog of Lemma 2.6, gives a sufficient condition for Schur multipliers that are not translation invariant (i.e., not of the form $M(t-s)$ ).

Lemma 2.8. Let $M \in W\left(\mathbb{R}^{N} \times \mathbb{R}^{N}\right)=W\left(\mathbb{R}^{2 N}\right)$. Then $M$ is a Schur multiplier on the set of restrictedly bounded singular kernels in $\mathbb{R}^{N}$ with Schur norm at most $\|M\|_{W\left(\mathbb{R}^{2 N}\right)}$.

Proof. Let $p, \mu$, and $\nu$ be fixed, and let $K$ be an $L^{p}$ restricted singular kernel on $\mathbb{R}^{N}$. Since for $a \in \mathbb{R}$ the multiplication by $e^{i a x}$ is an isometry in $L^{p}(\mu)$ (and in $\left.L^{p^{\prime}}(\nu)\right)$, the kernel $e^{-i a \cdot s} K(s, t) e^{-i b \cdot t}$ has the same $L^{p}$ restricted bound as the kernel $K$.

Because $\rho \in L^{1}\left(\mathbb{R}^{N} \times \mathbb{R}^{N}\right)$, one can immediately see that the restricted norm of the "averaged" kernel $\widetilde{K}$,

$$
\widetilde{K}(s, t):=\int_{\mathbb{R}^{N} \times \mathbb{R}^{N}} K(s, t) \rho(a, b) e^{-i a \cdot s} e^{-i b \cdot t} d a d b=K(s, t) \widehat{\rho}(s, t)
$$

is at most $\|\widehat{\rho}\|_{W}\|K\|_{\text {restr }}$, where $\|K\|_{\text {restr }}$ is the $\left(L^{p}, \mu, \nu\right)$ restricted norm of $K$.

Remark 2.9. In the above Lemma 2.8 one can replace class $W=W\left(\mathbb{R}^{N} \times \mathbb{R}^{N}\right)$ by the class $\widehat{\mathcal{M}}$ of the Fourier transforms of the measures (charges) of bounded variation in $\mathbb{R}^{N} \times \mathbb{R}^{N}$. 
This point of view unifies the situations described in Lemmas 2.6 and 2.8 . Namely, if one considers on the $N$-dimensional subspace $D=\left\{(-x, x): x \in \mathbb{R}^{N}\right\}$ the measure (not necessarily nonnegative) $\sigma, d \sigma=\rho(x) d x$ in the parametrization $x \mapsto(-x, x), x \in \mathbb{R}^{N}$, then the Fourier transform $\widehat{\sigma}$ of $\sigma$ (treated as a measure on the whole $\left.\mathbb{R}^{N} \times \mathbb{R}^{N}\right)$ is exactly $\widehat{\rho}(t-s)$.

Remark 2.10. One can use Lemma 2.7 for a sufficient condition for $M \in W\left(\mathbb{R}^{N} \times\right.$ $\left.\mathbb{R}^{N}\right)$; in this case it is sufficient that $M \in W^{k, 2}\left(\mathbb{R}^{2 N}\right), k>N$.

Remark. As we already mentioned, the Wiener algebra is scale invariant, i.e., the functions $M$ and $M_{\varepsilon}, M_{\varepsilon}(x):=M(x / \varepsilon)$ have the same norm in the Wiener algebra (a well known fact and an easy exercise). The same, of course, holds for the space $\widehat{\mathcal{M}}$.

Therefore, if $M \in \widehat{\mathcal{M}}$, and $M_{\varepsilon}(x):=M(x / \varepsilon)$, then all $M_{\varepsilon}$ are Schur multipliers with the uniform estimate on the Schur norm.

This fact will be exploited a lot in this paper.

Let us now state a simple corollary.

Corollary 2.11. Let $m$ be in the Sobolev space $H^{k}\left(\mathbb{R}^{N}\right), k>N / 2$. Then the kernels $K_{\varepsilon}, K_{\varepsilon}(s, t):=m((t-s) / \varepsilon)$ are Schur multipliers with uniformly bounded Schur norms.

\subsection{Smooth mollifying multipliers}

We can summarize the above discussion in the following proposition:

Proposition 2.12. Let $m$ be a function on $\mathbb{R}^{N}$ such that $m \equiv 0$ in a neighborhood of 0 , and $1-m \in H^{k}\left(\mathbb{R}^{N}\right)=W^{k, 2}\left(\mathbb{R}^{N}\right), k>N / 2$. Then the functions $M_{\varepsilon}$,

$$
M_{\varepsilon}(s, t):=m((t-s) / \varepsilon)
$$

are the family of smooth regularized multipliers, described above in Section 1.2, meaning that

(i) $M_{\varepsilon}(s, t) \rightarrow 1$ as $\varepsilon \rightarrow 0$ uniformly on all sets $\left\{s, t \in \mathbb{R}^{N}:|s-t|>a\right\}, a>0$;

(ii) for any singular kernel $K$ (with respect to Radon measures $\mu$ and $\nu$ ) the regularized kernels $K_{\varepsilon}=K M_{\varepsilon}$ are locally in $L^{2}(\mu \times \nu)$, so the corresponding operators are well defined for bounded compactly supported functions;

(iii) if the kernel is restrictedly bounded in $L^{p}$ (i.e., if the estimate (1.5) holds for all bounded $f, g$ with separated compact supports), and the measures $\mu$ and $\nu$ do not have common atoms, then the regularized integral operators $T_{\varepsilon}$ with kernels $K_{\varepsilon}$ are uniformly (in $\varepsilon$ ) bounded as operators $L^{p}(\mu) \rightarrow L^{p}(\nu)$. 


\section{Restricted boundedness implies boundedness}

In this section we are going to show that for kernels which are locally $L^{2}(\mu \times \nu)$ (and also for nonnegative kernels), restricted $L^{p}$ boundedness with restricted norm $C$ implies boundedness with norm at most $2 C$.

The main application of this result is as follows. Suppose $K$ is an $L^{p}$ restrictedly bounded singular kernel. Multiplying $K$ by a smooth mollifying multiplier, as described above in Section 2 (see for example Proposition 2.12), we will get a family of uniformly (in $\varepsilon$ ) restrictedly bounded kernels $K_{\varepsilon}:=M_{\varepsilon} K$. The kernels $K_{\varepsilon}$ are locally $L^{2}(\mu \times \nu)$, so, by the main result of this section, the corresponding operators $T_{\varepsilon}$ are uniformly bounded operators from $L^{p}(\mu)$ to $L^{p}(\nu)$.

Let $T$ be a limit point of $T_{\varepsilon}$, as $\varepsilon \rightarrow 0$, in the weak operator topology. Statement (i) of Proposition 2.12 will imply that for $f$ and $g$ with separated compact supports

$$
\langle T f, g\rangle_{\nu}=\int K(s, t) f(t) g(s) d \mu(t) d \nu(s)
$$

so the limit operator $T$ can indeed be considered as a singular integral operator with kernel $K$.

The statement about nonnegative kernels will be used to show that under some additional assumptions about the kernel $K$ (which do not involve nonnegativity), the restricted boundedness of $K$ implies that the measures $\mu$ and $\nu$ satisfy the two weight Muckenhoupt condition; see Theorem 5.1 below.

\subsection{Separated partitions of unity}

Lemma 3.1. Let $\sigma$ be a Radon measure without atoms on $\mathbb{R}^{N}$. There exist Borel sets $E_{n}^{1}, E_{n}^{2}, n \in \mathbb{N}$ such that

(i) For all $n \in \mathbb{N}$ the sets $E_{n}^{1}$ and $E_{n}^{2}$ are separated, i.e., $\operatorname{dist}\left(E_{n}^{1}, E_{n}^{2}\right)>0$.

(ii) The operators $P_{n}^{k}, P_{n}^{k} f:=\mathbf{1}_{E_{n}^{k}} f, k=1,2$ converge to $\frac{1}{2} I$ in the weak operator topology in $L^{2}(\sigma)$.

(iii) For any $p \in[1, \infty)$ and for $k=1,2$

$$
\lim _{n \rightarrow \infty}\left\|\mathbf{1}_{E_{n}^{k}} f\right\|_{L^{p}(\sigma)}=2^{-1 / p}\|f\|_{L^{p}(\sigma)}, \quad \forall f \in L^{p}(\sigma) .
$$

Definition. The standard grid $G$ of size $r$ in $\mathbb{R}^{N}$ is the collection of cubes $r \mathbf{j}+$ $[0, r)^{N}, \mathbf{j} \in \mathbb{Z}^{N}$. A grid of size $r$ is a translation of the standard grid by some $a \in \mathbb{R}^{N}$.

The boundary $\partial G$ of a grid $G$ is the union of all boundaries $\partial Q, Q \in G$.

For a cube $R=x+[0, r)^{N}$, with $x \in \mathbb{R}^{N}$ and $\tau \in \mathbb{R}$, let $\tau R$ denote its dilation, $\tau R:=x+[0, \tau r)^{N}$. Note that the cube is dilated with respect to its corner, not its center. 
Lemma 3.2. Let $\sigma$ be a Radon measure on $\mathbb{R}^{N}$. For a cube $R=x+[0, r)^{N}$ let $\tau R$ be its dilation as described above. Then,

$$
\lim _{\tau \rightarrow 1} \sigma(\tau R)=\sigma(R) .
$$

Proof. The lemma is a trivial corollary of countable additivity. Recall that for finite measures countable additivity is equivalent to the fact that $\sigma\left(\cup_{k \geq 0} R_{k}\right)=$ $\lim _{k \rightarrow \infty} \sigma\left(R_{k}\right)$ for any increasing sequence of measurable sets $R_{k}$. Since the family $\tau R, \tau>0$, is an increasing (with respect to $\tau$ ) family of cubes, and since $\cup_{\tau \in(0,1)} \tau R=R$, we get that

$$
\lim _{\tau \rightarrow 1-} \sigma(\tau R)=\sigma(R) .
$$

Since in what follows we only need this identity, we leave the rest of the lemma (i.e., the case of $\lim _{\tau \rightarrow 1+}$ ) as an easy exercise for the reader, who just needs to recall the restatement of countable additivity in terms of decreasing sequences of sets.

Proof of Lemma 3.1. Since for any sets $E_{n}^{k}$ the operators $P_{n}^{k}, P_{n}^{k} f:=\mathbf{1}_{E_{n}^{k}} f$, are contractions on all $L^{p}(\sigma)$, to prove (ii) it is sufficient to show that

$$
\lim _{n \rightarrow \infty}\left\langle P_{n}^{k} f, g\right\rangle=\frac{1}{2}\langle f, g\rangle
$$

for $f$ and $g$ in some set dense in $L^{p}(\sigma)$. In particular, it is sufficient to prove this identity for $f$ and $g$ that are finite sums $\sum_{j} \alpha_{j} \mathbf{1}_{Q_{j}}$, where $Q_{j}$ are some (standard) dyadic cubes.

Because of the continuity of the norm, it is also sufficient to check the condition (iii) on a dense set, for example again on the finite sums $\sum_{j} \alpha_{j} \mathbf{1}_{Q_{j}}$.

So, it is sufficient to show that for any standard dyadic cube $Q$

$$
\lim _{n \rightarrow \infty} \sigma\left(E_{n}^{k} \cap Q\right)=\frac{1}{2} \sigma(Q), \quad k=1,2 .
$$

To prove (3.1) we will construct the sets $E_{n}^{k}$ in such a way that

$$
\left|\sigma\left(E_{n}^{k} \cap Q\right)-\sigma(Q) / 2\right|<2^{-n} \sigma(Q), \quad k=1,2,
$$

for all standard dyadic cubes of size $2^{-n}$ which are inside the large cube $Q^{n}:=$ $\left[-2^{n}, 2^{n}\right)^{N}$. Trivially, the same estimate will hold for all larger dyadic cubes inside $Q^{n}$, which trivially implies (3.1).

Let us construct the sets $E_{n}^{k}$. For each $n$ we will first construct the sets $\widetilde{E}_{n}^{k}$, such that the sets $\widetilde{E}_{n}^{1}$ and $\widetilde{E}_{n}^{2}$ are disjoint (but not necessarily separated), and (3.2) is satisfied. The sets $\widetilde{E}_{n}^{k}$ will be constructed as unions of the (small) standard dyadic cubes, and by shrinking each cube a little, we will get separated sets $E_{n}^{1}$ and $E_{n}^{2}$.

Let $\alpha=\min _{Q} \sigma(Q)$, where the minimum is taken over all standard dyadic cubes $Q \subset Q^{n}$ of size $2^{-n}$ for which $\sigma(Q) \neq 0$. Pick $\delta_{0}>0$ such that

$$
\sigma(R)<2^{-n} \alpha
$$


for all cubes $R \subset Q^{n}$ such that $\ell(R)<\delta_{0}$ (recall, that $\ell(R)$ is the size, i.e., the sidelength of the cube $R$ ). Note, that clearly $\delta_{0}<2^{-n}$. Pick some $\delta<\delta_{0}$ of the form $\delta=2^{-m}, m \in \mathbb{Z}$.

Let us split the cube $Q^{n}$ into the standard dyadic cubes of size $\delta$, and construct the sets $\widetilde{E}_{n}^{1}$ and $\widetilde{E}_{n}^{2}$ as the finite unions of such cubes. Namely, for each dyadic cube $Q \subset Q^{n}, \ell(Q)=2^{-n}$, we distribute the dyadic cubes $R \subset Q, \ell(R)=\delta$, between the sets $\widetilde{E}_{n}^{1}$ and $\widetilde{E}_{n}^{2}$ as follows.

We assign the first such cube $R$ to be in $\widetilde{E}_{n}^{1}$, the second one to be in the set $\widetilde{E}_{n}^{2}$, and on each subsequent step we add a cube to the set of smaller measure $\sigma$ (in the case when both sets have the same measure, we can add the next cube to either of the sets, say to $\widetilde{E}_{n}^{1}$ for definiteness). We stop when all such cubes $R$ are exhausted, and then repeat the procedure for the other cubes $Q$.

Since, by the choice of $\delta$, for each dyadic cube $Q \subset Q^{n}, \ell(Q)=2^{-n}$, we have

$$
\sigma(R)<2^{-n} \alpha \leq 2^{-n} \sigma(Q)
$$

and since on each step we add such a cube $R$ to a set of smaller (or equal) measure, we conclude that

$$
\left|\sigma\left(\widetilde{E}_{n}^{1} \cap Q\right)-\sigma\left(\widetilde{E}_{n}^{2} \cap Q\right)\right|<2^{-n} \sigma(Q),
$$

which in turn implies (because $\left.\sigma\left(\widetilde{E}_{n}^{1} \cap Q\right)+\sigma\left(\widetilde{E}_{n}^{2} \cap Q\right)=\sigma(Q)\right)$ that for $k=1,2$,

$$
\left|\sigma\left(\widetilde{E}_{n}^{k} \cap Q\right)-\sigma(Q) / 2\right|<2^{-n-1} \sigma(Q) .
$$

We then obtain the sets $E_{n}^{1}$ and $E_{n}^{2}$ by replacing each dyadic cube $R, \ell(R)=\delta$, in the sets $\widetilde{E}_{n}^{1}$ and $\widetilde{E}_{n}^{2}$ by the cube $\tau R$, where $\tau \in(0,1)$ is sufficiently close to 1 . Clearly, for any $\tau \in(0,1)$ the sets $E_{n}^{1}$ and $E_{n}^{2}$ are separated. Moreover, Lemma 3.2 ensures that by picking $\tau$ sufficiently close to 1 we can make the differences $\sigma\left(\widetilde{E}_{n}^{k}\right)-$ $\sigma\left(E_{n}^{k}\right)$ as small as we want, so if $\tau$ is sufficiently close to $1,(3.2)$ holds.

Let us now consider the general case. For a measure $\mu$ let $\mu_{\mathrm{c}}$ and $\mu_{\mathrm{a}}$ be the continuous and purely atomic parts of $\mu$ respectively, $\mu=\mu_{\mathrm{c}}+\mu_{\mathrm{a}}$. For a $\mu$-measurable function $f$ consider the decomposition $f=f_{\mu_{\mathrm{c}}}+f_{\mu_{\mathrm{a}}}$, where $f_{\mu_{\mathrm{c}}}$ and $f_{\mu_{\mathrm{a}}}$ are the projections of $f$ onto the continuous and atomic parts of $\mu$ respectively,

$$
f_{\mu_{\mathrm{a}}}(x)= \begin{cases}f(x), & \mu(\{x\})>0, \\ 0, & \mu(\{x\})=0 .\end{cases}
$$

Corollary 3.3. Let $\mu$ and $\nu$ be Radon measures in $\mathbb{R}^{N}$ without common atoms. There exist Borel sets $E_{n}^{1}, E_{n}^{2}, n \in \mathbb{N}$ such that

(i) For all $n \in \mathbb{N}$ the sets $E_{n}^{1}$ and $E_{n}^{2}$ are separated, i.e., $\operatorname{dist}\left(E_{n}^{1}, E_{n}^{2}\right)>0$.

(ii) The operators $P_{n}^{1}$ and $P_{n}^{2}$, given by $P_{n}^{1} f:=\mathbf{1}_{E_{n}^{1}}\left(f_{\mu_{\mathrm{c}}}+\frac{1}{2} f_{\mu_{\mathrm{a}}}\right)$ and $P_{n}^{2} g:=$ $\mathbf{1}_{E_{n}^{2}}\left(g_{\nu_{\mathrm{c}}}+\frac{1}{2} g_{\nu_{\mathrm{a}}}\right)$ converge to $\frac{1}{2} I$ in the weak operator topology on $L^{2}(\mu)$ and $L^{2}(\nu)$, respectively.

(iii) For any $p \in[1, \infty)$ and for any $f \in L^{p}(\mu), g \in L^{p}(\nu)$

$$
\lim _{n \rightarrow \infty}\left\|\mathbf{1}_{E_{n}^{1}} f\right\|_{L^{p}(\mu)} \leq 2^{-1 / p}\|f\|_{L^{p}(\mu)}, \quad \lim _{n \rightarrow \infty}\left\|\mathbf{1}_{E_{n}^{2}} g\right\|_{L^{p}(\nu)} \leq 2^{-1 / p}\|g\|_{L^{p}(\nu)} .
$$


Proof. Take $\sigma:=\mu_{\mathrm{c}}+\nu_{\mathrm{c}}$, where $\mu_{\mathrm{c}}$ and $\nu_{\mathrm{c}}$ are the continuous parts of the measures $\mu$ and $\nu$, respectively. Then

$$
d \mu_{\mathrm{c}}=w d \sigma, \quad d \nu_{c}=v d \sigma,
$$

where $w, v \geq 0$ are some weights such that $w+v \equiv 1$.

If the measures $\mu$ and $\nu$ do not have atoms, then the sets $E_{n}^{1}$ and $E_{n}^{2}$ from Lemma 3.1 above are exactly the sets we need. Indeed, for bounded functions $f$ and $g$, statements (ii) and (iii) of the corollary (weak convergence and convergence of norms in $L^{p}(\mu)$ and $L^{p}(\nu)$ ) follow from the corresponding statements of Lemma 3.1 (convergence in $L^{p}(\sigma)$ ). Since the operators $P_{n}^{k}$ are contractions for any choice of the sets $E_{n}^{k}$, one can use the $\varepsilon / 3$-theorem to extend the statements (ii) and (iii) from a dense set of bounded functions to all of $L^{p}(\mu)\left(L^{p}(\nu)\right)$.

For the general case, let

$$
\mu_{\mathrm{a}}=\sum_{n} \alpha_{n} \delta_{x_{n}} \quad \text { and } \quad \nu_{\mathrm{a}}=\sum_{n} \beta_{n} \delta_{y_{n}}
$$

be the purely atomic parts of the measures $\mu$ and $\nu$, respectively. Without loss of generality, assume that the sequences $\left\{\alpha_{n}\right\}$ and $\left\{\beta_{n}\right\}$ are non-increasing. Note that since the measures $\mu$ and $\nu$ do not have a common atom, $x_{n} \neq y_{k}$ for all $n$ and $k$.

Let $\widetilde{E}_{n}^{1}$ and $\widetilde{E}_{n}^{2}$ denote the sets obtained in Lemma 3.1 for $\sigma:=\mu_{\mathrm{c}}+\nu_{\mathrm{c}}$ (we use the notation $\widetilde{E}_{n}^{k}$ instead of $E_{n}^{k}$ because we reserve the notation $E_{n}^{k}$ for the final "output"). We can always assume without loss of generality that $x_{j}, y_{j} \notin \widetilde{E}_{n}^{k}$ (for all $j, n$ and $k$ ). Define

$$
E_{n}^{1}:=\left(\widetilde{E}_{n}^{1} \cup \bigcup_{j=1}^{n} x_{j}\right) \backslash \bigcup_{j=1}^{\infty} B\left(y_{j}, r_{j}^{(n)}\right), \quad E_{n}^{2}:=\left(\widetilde{E}_{n}^{2} \cup \bigcup_{j=1}^{n} y_{j}\right) \backslash \bigcup_{j=1}^{\infty} B\left(x_{j}, r_{j}^{(n)}\right),
$$

where for each $n$ the radii $r_{j}^{(n)}$ are picked in such a way that

$$
\sum_{j=1}^{\infty}\left[\sigma\left(B\left(x_{j}, r_{j}^{(n)}\right)\right)+\sigma\left(B\left(y_{j}, r_{j}^{(n)}\right)\right)\right]<2^{-n}
$$

and such that

$$
\bigcup_{j=1}^{n} x_{j} \cap \bigcup_{j=1}^{\infty} B\left(y_{j}, r_{j}^{(n)}\right)=\bigcup_{j=1}^{n} y_{j} \cap \bigcup_{j=1}^{\infty} B\left(x_{j}, r_{j}^{(n)}\right)=\varnothing .
$$

Let us show that the $E_{n}^{k}$ are the desired sets. Let us decompose $f \in L^{p}(\mu)$ as $f=f_{\mathrm{c}}+f_{\mathrm{a}}$, where $f_{\mathrm{a}}:=f \mathbf{1}_{\cup_{j=1}^{\infty}\left\{x_{j}\right\}}$ is the purely atomic part of $f$. Clearly

$$
\lim _{n \rightarrow \infty}\left\|f_{\mathrm{a}}-\mathbf{1}_{E_{n}^{1}} f_{\mathrm{a}}\right\|_{L^{p}(\mu)}=0
$$

so statements (ii) and (iii) of the corollary hold for purely atomic functions $f_{\mathrm{a}}$. Note that, unlike in Lemma 3.1, we have here inequality in statement (iii), because

$$
\lim _{n \rightarrow \infty}\left\|2^{-1} \mathbf{1}_{E_{n}^{1}} f_{\mathrm{a}}\right\|_{L^{p}(\mu)}=2^{-1}\left\|f_{\mathrm{a}}\right\|_{L^{p}(\mu)} \leq 2^{-1 / p}\left\|f_{\mathrm{a}}\right\|_{L^{p}(\mu)} .
$$


We can also estimate

$$
\left\|f_{\mathrm{c}} \mathbf{1}_{E_{n}^{1}}-f_{\mathrm{c}} \mathbf{1}_{\widetilde{E}_{n}^{1}}\right\|_{L^{p}\left(\mu_{\mathrm{c}}\right)}^{p} \leq \int_{\bigcup_{j=1}^{\infty} B\left(y_{j}, r_{j}^{(n)}\right)}\left|f_{\mathrm{c}}\right|^{p} d \mu_{\mathrm{c}} \rightarrow 0 \quad \text { as } n \rightarrow \infty .
$$

because

$$
\mu_{\mathrm{c}}\left(\bigcup_{j=1}^{\infty} B\left(y_{j}, r_{j}^{(n)}\right)\right) \leq \sigma\left(\bigcup_{j=1}^{\infty} B\left(y_{j}, r_{j}^{(n)}\right)\right) \leq 2^{-n}
$$

Therefore, since statements (ii) and (iii) of the corollary hold for the sets $\widetilde{E}_{n}^{1}$ and the measure $\mu_{\mathrm{c}}$ (because, as we discussed above, the sets from Lemma 3.1 work for measures without atoms), equality (3.4) implies that these statements hold for the sets $E_{n}^{1}$ and the measure $\mu_{\mathrm{c}}$ as well.

So statements (ii) and (iii) of the corollary hold for $f_{\mathrm{a}}$ and $f_{\mathrm{c}}$ (and the measure $\mu$ ), and therefore they are true for $f$.

The statements for the measure $\nu$ can be proved in exactly the same way.

\subsection{Uniform boundedness}

Theorem 3.4. Let $\mu$ and $\nu$ be Radon measures on $\mathbb{R}^{N}$ without common atoms. Assume that a kernel $K \in L_{\mathrm{loc}}^{2}(\mu \times \nu)$ is $L^{p}$ resrictedly bounded, with the restricted norm $C$. Then the integral operator $T$ with kernel $K$ is a bounded operator $L^{p}(\mu) \rightarrow$ $L^{p}(\nu)$ with norm at most $2 C$.

Proof. Let $f$ and $g$ be (Borel measurable) functions, supported on a cube $Q$. Let us restrict everything to the cube $Q$. Then the integral operator $T$ with kernel $K$ is a Hilbert-Schmidt (and therefore compact) operator, $T: L^{2}(Q, \mu) \rightarrow L^{2}(Q, \nu)$.

Let $P_{n}^{k}$ be the projections from Corollary 3.3. Then by statement (ii) of the corollary (weak convergence) and because $T$ is compact,

$$
\lim _{n \rightarrow \infty}\left\langle T P_{n}^{1} f, P_{n}^{2} g\right\rangle=\frac{1}{4}\langle T f, g\rangle .
$$

On the other hand, by restricted $L^{p}$ boundedness

$$
\left|\left\langle T P_{n}^{1} f, P_{n}^{2} g\right\rangle\right| \leq C\left\|P_{n}^{1} f\right\|_{L^{p}(\mu)}\left\|P_{n}^{2} g\right\|_{L^{p^{\prime}}(\nu)}
$$

and by statement (iii) of Corollary 3.3

$$
\lim _{n \rightarrow \infty}\left\|P_{n}^{1} f\right\|_{L^{p}(\mu)}\left\|P_{n}^{2} g\right\|_{L^{p^{\prime}(\nu)}}=2^{-1 / p}\|f\|_{L^{p}(\mu)} 2^{-1 / p^{\prime}}\|g\|_{L^{p^{\prime}(\nu)}} .
$$

So, taking the limit on both sides of (3.5) we get that

$$
\frac{1}{4}|\langle T f, g\rangle| \leq \frac{1}{2}\|f\|_{L^{p}(\mu)}\|g\|_{L^{p^{\prime}}(\nu)} .
$$

which is exactly the desired estimate. Since it holds on a dense set of bounded compactly supported functions, we are done. 
The next result is an easy corollary of the previous theorem.

Theorem 3.5. Let $\mu$ and $\nu$ be Radon measures on $\mathbb{R}^{N}$ without common atoms. Assume that a kernel $K \geq 0$ is $L^{p}$ resrictedly bounded, with the restricted norm $C$. Then the integral operator $T$ with kernel $K$ is a bounded operator $L^{p}(\mu) \rightarrow L^{p}(\nu)$ with norm at most $2 C$.

Proof. First, notice that the integral operator with kernel $K \geq 0$ is well defined for $f \geq 0$, and to compute its norm we only need to test it on $f \geq 0$.

Second, the norm of this operator can be computed as the supremum (or limit as $R \rightarrow \infty$ ) of the operators with the truncated kernels

$$
K_{R}(s, t)=\min \{K(s, t), R\}, \quad R>0 .
$$

If $C$ is the restricted norm of $K$, then $C$ is also a restricted bound for all of the $K_{R}$. However, the kernels $K_{R}$ are bounded, so by Theorem 3.4 the corresponding integral operators are bounded with norm at most $2 C$. Taking the limit as $R \rightarrow \infty$ we get the conclusion of the theorem.

\subsection{How to treat common atoms}

If the measures $\mu$ and $\nu$ do have common atoms, then the above Theorem 3.4 cannot be applied directly. However, using this theorem, it is quite easy to define the boundedness of the singular integral operator in this case.

Namely, consider the decompositions

$$
\mu=\widetilde{\mu}+\mu_{0} \quad \text { and } \quad \nu=\widetilde{\nu}+\nu_{0},
$$

where $\mu_{0}$ and $\nu_{0}$ are the parts of $\mu$ and $\nu$ supported on their common atoms. Then the measures $\mu$ and $\widetilde{\nu}$ do not have common atoms; the same is true for $\widetilde{\mu}$ and $\mu$. Therefore, we check the $L^{p}$ boundedness of a singular integral operator with kernel $K$ as an operator $L^{p}(\mu) \rightarrow L^{p}(\widetilde{\nu})$ or $L^{p}(\widetilde{\mu}) \rightarrow L^{p}(\nu)$. But since the measures do not have common atoms, these operators can be checked using Theorem 3.4.

So, to check the boundedness of the whole operator, it remains to check the block mapping $L^{p}\left(\mu_{0}\right) \rightarrow L^{p}\left(\nu_{0}\right)$. But the bilinear form corresponding to this block is well defined for functions supported at finitely many points (note, that the kernel $K(x, x)$ has to be defined at common atoms of the measures $\mu$ and $\nu$ ), so there is no problem defining this block.

\section{Uniform boundedness of truncations}

In this section we will show that under some additional assumptions, which are satisfied for classical operators like the generalized Riesz transforms (treated as a vector-valued transformation), or the Ahlfors-Beurling operator, restricted $L^{p}$ boundedness implies uniform boundedness of the truncated operators $T_{\varepsilon}$,

$$
T_{\varepsilon} f(s)=\int_{|s-t|<\varepsilon} K(s, t) f(t) d \mu(t) .
$$


We will need the following definition:

Definition 4.1. Let $\kappa \in(0,1]$. A function $F$ with values in $\mathbb{R}^{d}$ is called $\kappa$-sectorial if there exists $x_{0} \in \mathbb{R}^{d},\left|x_{0}\right|=1$, such that $\left\langle F(s), x_{0}\right\rangle \geq \kappa|F(s)|$ for all $s$ in the domain of $F$.

Proposition 4.2. Let a singular kernel $K$ with values in $\mathbb{R}^{k}$ be restrictedly bounded in $L^{p}$ (we assume that the measures $\mu$ and $\nu$ on $\mathbb{R}^{N}$ without common atoms have been fixed).

Suppose there exist $\delta>0, \kappa>0$ and a family of (matrix-valued) Schur multipliers $M_{\varepsilon}(s, t), \varepsilon \in(0, \infty)$, with uniformly bounded Schur norm, such that

(i) for each $\varepsilon>0$ the function $M_{\varepsilon} K$ is $\kappa$-sectorial on the set $\left\{s, t \in \mathbb{R}^{N}\right.$ : $(1-\delta) \varepsilon<|s-t|<\varepsilon\}$

(ii) $\left|M_{\varepsilon} K\right| \geq|K|$ on the set $\left\{s, t \in \mathbb{R}^{N}:(1-\delta) \varepsilon<|s-t|<\varepsilon\right\}$.

Then the truncated operators $T_{\varepsilon}$ defined by (4.1) are uniformly (in $\varepsilon$ ) bounded operators $L^{p}(\mu) \rightarrow L^{p}(\nu)$.

Proof. Take a function $m \in C^{\infty}(\mathbb{R})$ such that $m(x)=1$ for $x \geq 1$ and $m(x)=0$ for $x \leq 1-\delta$. Then the function $\tilde{m}, \widetilde{m}(s)=m(|s|), s \in \mathbb{R}^{N}$ satisfies $1-\widetilde{m} \in$ $C_{0}^{\infty}\left(\mathbb{R}^{N}\right)$. As was discussed in Section 2 above, this implies that the functions $m(|s-t| / \varepsilon)=\widetilde{m}((s-t) / \varepsilon)$ are Schur multipliers with a uniform (in $r$ ) estimate on the Schur norm.

Therefore, the smoothly regularized kernels $m(|s-t| / \varepsilon) K(s, t)$ give a uniformly bounded family of operators. The difference between the kernel of the truncated operator $T_{\varepsilon}$ and the kernel $m(|s-t| / \varepsilon) K(s, t)$ is given by $\psi(|s-t| / \varepsilon) K(s, t)$, where $\psi(x)=m(x)-\mathbf{1}_{[1, \infty)}(x)$. Thus, to prove the uniform boundedness of the truncated operators, it is sufficient to prove the uniform boundedness of the operators with kernels $\psi(|s-t| / \varepsilon) K(s, t)$.

We now use the trivial observation that if $T_{1}, T_{2}$ are integral operators between $L^{p}$ spaces (defined initially on dense sets) with kernels $K_{1}$ and $K_{2}$ respectively, and if $\left|K_{1}\right| \leq K_{2}$, then the boundedness of $T_{2}$ implies the boundedness of $T_{1}$ and the estimate $\left\|T_{1}\right\| \leq\left\|T_{2}\right\|$. Therefore, since

$$
|\psi(|s-t| / \varepsilon) K(s, t)| \leq \chi(|s-t| / \varepsilon)|K(s, t)|,
$$

where $\chi:=\mathbf{1}_{[1-\delta, 1]}$, to prove the proposition it is sufficient to show that the operators with kernels $\chi(|s-t| / \varepsilon)|K(s, t)|$ are uniformly (in $\varepsilon$ ) bounded.

Let $M_{\varepsilon}$ be the Schur multiplier from the assumption of the proposition, and let $x_{0} \in \mathbb{R}^{d}$ be such that

$$
\left\langle M_{\varepsilon}(s, t) K(s, t), x_{0}\right\rangle \geq \kappa|K(s, t)| \quad \forall s, t \in \mathbb{R}^{N}:(1-\delta) \varepsilon<|s-t|<\varepsilon .
$$

The operators with (vector-valued) kernels $M_{\varepsilon}(s, t) K(s, t)$ are uniformly (in $\varepsilon$ ) bounded, and therefore so are the operators with scalar-valued kernels given by $\left\langle M_{\varepsilon}(s, t) K(s, t), x_{0}\right\rangle$. 
The estimate (4.2) implies that

$$
\chi(|s-t| / \varepsilon)|K(s, t)| \leq \kappa^{-1}\left\langle M_{\varepsilon}(s, t) K(s, t), x_{0}\right\rangle,
$$

and thus the operators with the kernels $\chi(|s-t| / \varepsilon)|K(s, t)|$ are uniformly bounded.

\subsection{Some examples}

Example 4.3. Consider a convolution kernel $K(s, t)=K_{1}(t-s)$,

$$
K_{1}(x)=A(|x|) B(x /|x|),
$$

where $A(r) \geq 0$ for all $r>0$ and $B$ is a function (with values in some $\mathbb{R}^{m}$ ) in the Sobolev space $H^{k}, k>N / 2$ on the unit sphere $\mathbb{S}_{N-1}$ in $\mathbb{R}^{N}$.

If $B(s) \neq 0$ for all $s \in \mathbb{S}_{N-1}$, then the kernel $K$ satisfies the assumption of Proposition 4.2.

Indeed, let

$$
C=\max _{s \in \mathbb{S}_{N-1}}|B(s)|^{-1},
$$

and let $\varphi \in C_{0}^{\infty}(\mathbb{R})$ be such that $\varphi \equiv 1$ on $[0.9,1]$, and $\varphi(x) \equiv 0$ for $x \notin(0.8,1.1)$. Then the function $m, m(s)=C \varphi(s) B^{T}(s /|s|)$, where $B^{T}$ stands for the transpose of $B$, is clearly in $H^{k}\left(\mathbb{R}^{N}\right)$.

Therefore, by Corollary 2.11 the functions $M_{r}(s, t)$ defined by

$$
M_{r}(s, t):=m((t-s) / r)=C \varphi(|s-t| / r) B^{T}((t-s) /|t-s|)
$$

are Schur multipliers with uniformly bounded Schur norms. It is trivial to see that the assumptions of (i) and (ii) of Proposition 4.2 are also satisfied (with $\kappa=1$ and $\delta=0.1)$.

Thus, for any such kernel, restricted $L^{p}$ boundedness implies uniform boundedness of the truncated operators defined by (4.1).

Examples of such kernels include the kernel of the (vector-valued) generalized Riesz transform in $\mathbb{R}^{N}\left(K_{1}(s)=s /|s|^{\alpha+1}, s \in \mathbb{R}^{N}, \alpha>0\right)$, or the Cauchy $\left(K_{1}(z)=\right.$ $\left.1 / z=\bar{z} /|z|^{2}\right)$ and the Ahlfors-Beurling $\left(K_{1}(z)=1 / z^{2}=\bar{z}^{2} /|z|^{4}\right)$ transforms in the complex plane.

Note that the classical Riesz transform is a particular case $(\alpha=N)$ of the generalized one.

Another example is given by the Beurling-Ahlfors transform $\mathcal{S}$ on forms, defined, for example in [4]. The fact that the kernel of $\mathcal{S}$ admits the representation (4.3) can be easily seen from formula (112) on p. 53 of [4].

Remark 4.4. Notice that Proposition 4.2 cannot be applied to individual (coordinate) generalized Riesz transforms given by $K_{1}(s)=s_{k} /|s|^{\alpha+1}$, where $s=$ $\left(s_{1}, s_{2}, \ldots, s_{N}\right)^{T}$. While the restricted boundedness of such an individual kernel implies the uniform boundedness of its smooth regularizations, we do not know whether it implies the uniform boundedness of the truncations (4.3).

We suspect that the answer here is negative. 


\section{Two weight Muckenhoupt condition}

Theorem 5.1. Let $K$ be a (vector-valued) convolution kernel in $\mathbb{R}^{N}$, namely $K(s, t)=K_{1}(t-s)$,

$$
K_{1}(s)=A(|x|) B(x)
$$

where $B \in H^{k}\left(\mathbb{R}^{N}\right)=W^{k, 2}\left(\mathbb{R}^{N}\right), k>N / 2$ is a function homogeneous of order $d>0$,

$$
B(c x)=c^{d} B(x) \quad \forall x \in \mathbb{R}^{N} \forall c \in \mathbb{R}_{+},
$$

bounded away from 0 on the unit sphere, and $A$ is a function on $\mathbb{R}_{+}$such that, for some $\alpha \geq d$,

$$
A(x) \geq x^{-d-\alpha} \quad \forall x \in \mathbb{R}_{+} .
$$

Let $\mu$ and $\nu$ be Radon measures without common atoms on $\mathbb{R}^{N}$, and let the kernel $K$ be $L^{p}$ restrictedly bounded (with respect to the measures $\mu$ and $\nu$ ).

Then the measures $\mu$ and $\nu$ satisfy the following generalized two-weight Muckenhoupt $A_{p}^{\alpha}$ condition of order $\alpha$ :

$$
\sup _{B}(\operatorname{diam} B)^{-\alpha} \mu(B)^{1 / p^{\prime}} \nu(B)^{1 / p}<\infty .
$$

Here the supremum is taken over all balls in $\mathbb{R}^{N}$.

Examples of kernels satisfying the assumptions of this proposition are the generalized vector-valued Riesz transform $(d=1)$, the Cauchy transform in $\mathbb{C}(\alpha=1$, $d=1)$, and the Beurling-Ahlfors transform in $\mathbb{C}(\alpha=2, d=2)$.

The classical Muckenhoupt condition $(\alpha=N)$ is well known in analysis: for classical (one weight) weighted estimates $\left(d \mu=w d x, d \nu=w^{-1} d x\right)$ it is well known that the classical Muckenhoupt condition $A_{p}=\left(A_{p}^{N}\right)(\alpha=N)$ is necessary and sufficient for the $L^{p}$ boundedness of classical singular integral operators, like Hilbert transform, or vector Riesz transform.

In the case of one measure $(\mu=\nu)$ the condition $\left(A_{p}^{\alpha}\right)$ is independent of $p \in$ $(1, \infty)$ and is equivalent to the growth estimate $\mu\left(B\left(x_{0}, r\right)\right) \leq C r^{\alpha}$ (where $B\left(x_{0}, r\right)$ stands for the open ball of radius $r$ centered at $x_{0}$ ) uniformly in $x_{0}$ and $r$.

In the one measure case this growth condition for $\alpha=1$ is known to be necessary (but not sufficient) for the boundedness in $L^{2}(\mu)$ of the Cauchy transform in $\mathbb{C}$. It is probably well known to specialists, although we do not know a reference, that in the one measure case the growth condition $\mu\left(B\left(x_{0}, r\right)\right) \leq C r^{\alpha}$ is necessary for the boundedness of the vector Riesz transform in $L^{p}(\mu)$.

This condition has also appeared in more general situations as well. For example, it was shown by the second author (see [9], p. 318) that the condition $\left(A_{2}^{1}\right)$ (in fact, a bit stronger version, where averages over intervals are replaced by Poisson averages) is necessary for the boundedness of the Hilbert transform in the general two weight situation. As easy examples show, the two weight $A_{2}$ condition is not sufficient for the $L^{2}$ boundedness of the Hilbert transform in the general two weight case. 
It was conjectured for some time that the stronger "Poisson" $A_{2}$ condition (which is necessary for the two weight estimate of the Hilbert transform) is also sufficient for the two weight estimate, but this conjecture was later disproved by F. Nazarov.

However, the necessity of this condition for general operators as in the above theorem is completely new, and has not appeared in the literature. The only exception here is probably our paper [6], where it was shown that the condition $\left(A_{2}^{1}\right)$ is necessary for the $L^{2}$ boundedness of rather general singular integral operators $T: L^{2}(\mu) \rightarrow L^{2}(\nu)$ on the real line.

For example, in the general two weight case, even the necessity of the condition $\left(A_{2}^{1}\right)$ for the $L^{2}$ boundedness of the Cauchy transform was not known (at least it has not been described in the literature). The same can be said for the condition $\left(A_{p}^{2}\right)$ for the $L^{p}$ boundedness of the Beurling-Ahlfors transform in $\mathbb{C}$. The result for the generalized vector Riesz transforms of order $\alpha$ is also new.

Proof of Theorem 5.1. Define

$$
m(x):=B(x) \varphi(|x|), \quad \forall x \in \mathbb{R}^{N},
$$

where $\varphi \in C_{0}^{\infty}(\mathbb{R})$ is such that $\varphi \equiv 1$ on $[0,2]$. Clearly $m \in H^{k}\left(\mathbb{R}^{N}\right)=W^{k, 2}\left(\mathbb{R}^{N}\right)$, so by Corollary 2.11 the functions $M_{\varepsilon}, M_{\varepsilon}(s, t):=m((t-s) / \varepsilon)$ are Schur multipliers with uniformly bounded Schur norms.

Since $B(x)=|x|^{d} B\left(x /|x|^{d}\right) \geq C|x|^{d}$, we conclude that

$$
|B(x)| \geq C|x|^{d}
$$

where $C=\inf \left\{|B(x)|: x \in \mathbb{R}^{N},|x|=1\right\}>0$. Then we can estimate

$$
B^{T}(x / \varepsilon) B(x) \geq C^{2}|x|^{2 d} \varepsilon^{-d},
$$

so for $|s-t| \leq 2 \varepsilon$,

$$
K_{\varepsilon}(s, t):=M_{\varepsilon}^{T}(s, t) K(s, t) \geq C^{2} \varepsilon^{-d}|t-s|^{d-\alpha} \geq C^{\prime} \varepsilon^{-\alpha} .
$$

Here in the last inequality we used the fact that $\alpha \geq d$. Note that $K_{\varepsilon}(s, t) \geq 0$ for all $s, t \in \mathbb{R}^{N}$.

Since the $M_{\varepsilon}$ are uniformly bounded Schur multipliers, the operators $T_{\varepsilon}$ with kernels $K_{\varepsilon}$ are uniformly (in $\varepsilon$ ) restrictedly bounded, and so, by Theorem 3.5 they are uniformly bounded operators $L^{p}(\mu) \rightarrow L^{p}(\nu)$.

Let $\mathcal{B}=\mathcal{B}\left(t_{0}, \varepsilon\right)$ be the open ball of radius $\varepsilon$ centered at $t_{0}$, and let $T_{\varepsilon}$ be the integral operator with kernel $K_{\varepsilon}$. Then estimate (5.3) implies that

$$
T_{\varepsilon} \mathbf{1}_{\mathcal{B}}(s) \geq C^{\prime} \varepsilon^{-\alpha} \mu(\mathcal{B}) \quad \forall s \in \mathcal{B} .
$$

Then integrating over $\mathcal{B}$ we get

$$
\left\|T \mathbf{1}_{\mathcal{B}}\right\|_{L^{p}(\nu)} \geq C^{\prime} \varepsilon^{-\alpha} \mu(\mathcal{B}) \nu(\mathcal{B})^{1 / p} .
$$

Since

$$
\left\|\mathbf{1}_{\mathcal{B}}\right\|_{L^{p}(\mu)}=\mu(\mathcal{B})^{1 / p}
$$


and the operators $T_{\varepsilon}$ are uniformly bounded (as operators $L^{p}(\mu) \rightarrow L^{p}(\nu)$ ), we get the estimate

$$
\varepsilon^{-\alpha} \mu(\mathcal{B}) \nu(\mathcal{B})^{1 / p} \leq C \mu(\mathcal{B})^{1 / p},
$$

with $C$ independent of $\mathcal{B}$ and $\varepsilon$. This estimate is equivalent to the conclusion of the proposition.

\section{Concluding remarks}

The main result of this paper simplifies, even trivializes, the definition of a singular integral operator if only its kernel is given. This paper does not offer a replacement for the hard analysis techniques used to prove the boundedness of singular integral operators; one still has to do hard work to prove boundedness. However, it significantly simplifies the setup.

For example, in [8] the authors had to spend a lot of time and effort carefully defining their operators. While this was necessary to state the result in full generality, in all interesting situations the operators were abstract singular integral operators, meaning that there was a kernel kernel $K(s, t)$ locally bounded off the diagonal giving the bilinear form for functions with separated compact supports. For example, this approach would work for the so called dyadic (or Haar) shifts, which recently have attracted much attention, see [5] and [3].

But as we have shown in this paper, such operators can be regularized by smooth mollifying multipliers! That means that if the operator is restrictedly bounded, then its "smooth" regularizations are uniformly bounded, so from the very beginning we can deal with such regularizations.

It looks a bit ironic that while the kernels of such dyadic integral operators are very non-smooth, they can be regularized by smooth multipliers. It would be interesting to find regularizations better adapted to the dyadic structure of such operators.

Next, we should mention that since our Schur multipliers are the universal ones, our approach works for operators from $L^{p}$ to $L^{r}, r \neq p$, as well. We did not want to overload the paper, so we only considered the case $p=r$ in the text. However, the corresponding general statements and their proofs can be easily obtained from the corresponding parts in the text by obvious modifications.

We should also mention that in the classical situation $(d \mu=d \nu=d x)$, and even in the "classical non-homogeneous" (one measure) situation $(d \mu=d \nu)$, it is known that if we have an operator $T$ bounded in some $L^{p}$ and having CalderónZygmund kernel $K$ (meaning that the bilinear form for functions with separated compact supports is given by $\left.\int K(s, t) f(t) g(s) d \mu(t) d \nu(s)\right)$, then its truncations $T_{\varepsilon}$ are uniformly bounded, and, moreover, the maximal operator $T^{\sharp}$ is also bounded.

However, this is a very non-trivial result, requiring rather strong assumptions (Calderón-Zygmund kernels, restrictions on the growth of the measure, even in the non-homogeneous case). No analogues of our results for more general situations (two measures, no restriction on the growth) were known before. Moreover, we suspect that the statement about the maximal operator $T^{\sharp}$ fails in the general situation. 


\section{References}

[1] Christ, M.: Lectures on singular integral operators. CBMS Regional Conference Series in Mathematics 77. Published for the Conference Board of the Mathematical Sciences, Washington, DC, American Mathematical Society, Providence, RI, 1990.

[2] Grafakos, L.: Modern Fourier analysis. Second edition. Graduate Texts in Mathematics 250, Springer, New York, 2009.

[3] Hytönen, T., Pérez, C., Treil, S. And Volberg, A.: Sharp weighted estimates of the dyadic shifts and $A_{2}$ conjecture. To appear in J. Reine Angew. Math., DOI: 10.1515/crelle-2012-0047, May 2012.

[4] Iwaniec, T. And Martin, G.: Riesz transforms and related singular integrals. J. Reine Angew. Math. 473 (1996), 25-57.

[5] Lacey, M., Petermichl, S. And Reguera, M.: Sharp $A_{2}$ inequality for haar shift operators. Math. Ann. 348 (2010), no. 1, 127-141.

[6] Liaw, C. AND Treil, S.: Rank one perturbations and singular integral operators. J. Funct. Anal. 257 (2009), no. 6, 1947-1975.

[7] Nazarov, F., Treil, S. and Volberg, A.: The Tb-theorem on non-homogeneous spaces. Acta Math. 190 (2003), no. 2, 151-239.

[8] Nazarov, F., Treil, S. And Volberg, A.: Two weight inequalities for individual Haar multipliers and other well localized operators. Math. Res. Lett. 15 (2008), no. $3,583-597$.

[9] Havin, V. P. and Nikolski, N. K. (eds.): Linear and complex analysis. Problem book 3. Part I. Lecture Notes in Mathematics 1573, Springer-Verlag, Berlin, 1994.

[10] Stein, E.: Harmonic analysis: real-variable methods, orthogonality, and oscillatory integrals. Princeton Mathematical Series 43, Monographs in Harmonic Analysis III, Princeton University Press, Princeton, NJ, 1993.

Received January 26, 2011.

Constanze Liaw: Department of Mathematics, Baylor University, One Bear Place 97328, Waco, TX 76798-7328, USA.

E-mail: constanze_liaw@baylor.edu

Sergei Treil: Department of Mathematics, Brown University, 151 Thayer Str./Box 1917, Providence, RI 02912, USA.

E-mail: treil@math.brown.edu 